\title{
Undescended testis with congenital unilateral absence of the vas deferens
}

\author{
V.D. Kulkarni, N.A. Deshpande, P.N. Deshpande, M. Phanse
}

Department of Surgery, Y.C.M.Hospital, Pimpri Pune, India.

Keywords: Vas deferens; cryptorchidism; cystic fibrosis.

\begin{abstract}
Congenital absence of the vas deferens (CAVD) is a condition in which the vasa deferentia, fail to form properly prior to birth. It may either be unilateral (CUAVD) or bilateral (CBAVD). Congenital absence of vas deferens is responsible for upto $2 \%$ of male infertility. The disease is considered to be a primary genital form of cystic fibrosis. It contributes towards $6 \%$ of obstructive azoospermia cases. Upto $80 \%$ of patients with CAVD have mutations accociated with Cystic Fibrosis Transmembrane Conductance (CFTR) gene. Upper urinary tract malformations are noted in upto $20 \%$ of cases. This category of patients have a normal frequency of CFTR mutations and considered a distinct clinical entity. We hereby report a rare case of CUAVD with ipsilateral renal agenesis with cryptorchidism.
\end{abstract}

\section{Introduction}

Disturbances in the development of human embryo are responsible for the absence of vas deferens. Vas deferens is derived from the mesonephric duct while the testis and the globus major of the epididymis arise from the genital ridge. The extent of abnormalities depends on the time at which mesonephric duct stops developing. If it occurs early in embryonic life (around the fourth week) the ureter and kidney are absent [1]. If the disturbances happen beyond fourth week, the kidney and ureter are present, although their development could be abnormal [2] .

\section{Case History}

A 12 year old child presented with non-descent of the right testis since birth. He did not have associated abdominal pain. On examination, the right testis was

Correspondence: V.D. Kulkarni

E-mail: drvdkulkarni@gmail.com neither palpable in the scrotum nor found in the right inguinal region. The left testis was normal in size and the cord was palpable on scrotal examination. There was no report of hospital admissions in the past. Ultrasonography of the abdomen revealed an absent right kidney, the left kidney being normal in size and shape. The right testis was detected, by ultrasound, in the right inguinal region. Intravenous pyelography confirmed the above findings (Figure 1). Renal function tests were normal. Sweat sodium estimation was in the normal range. The patient received an orchidopexy after informed consent was obtained from his parents. Intraoperatively the right testis was in inguinal canal ( Figure 2). On evaluating cord structures, the vas deferens was absent. Following orchidopexy, he was discharged on the second day.

\section{Discussion}

Abnormalities in the vas deferens depend on the time at which the mesonephric duct stops developing. Renal agenesis associated with CUAVD occurs if the

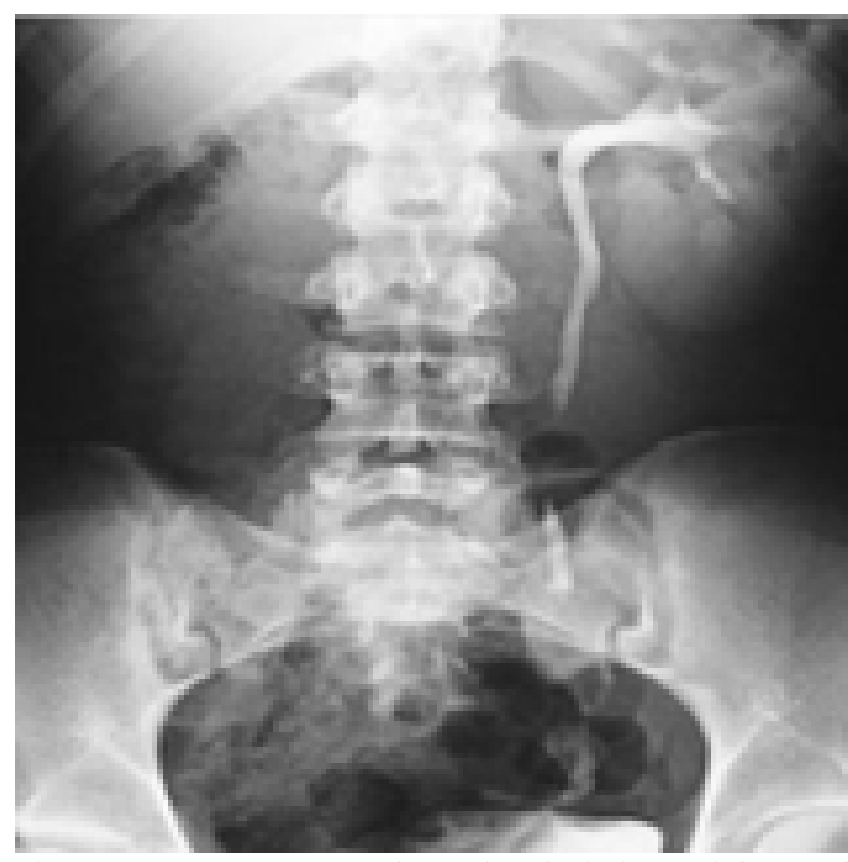

Figure 1. Intravenous pyelography depicting right renal agenesis 


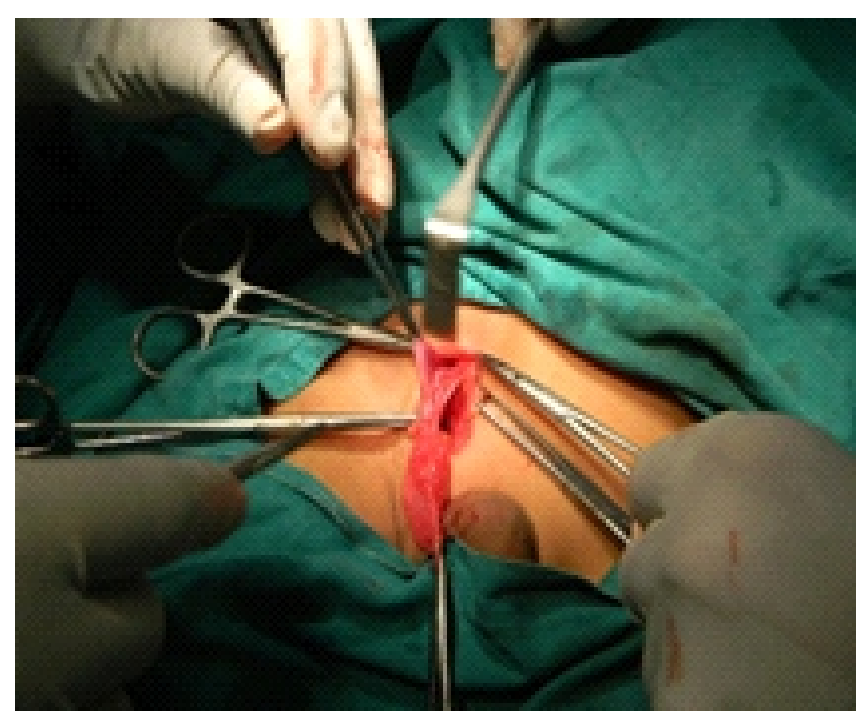

Figure 2. Right testis in the inguinal canal

mesonephric duct stops developing by the fourth week [1]. The vas deferens is a cord like structure which can be palpated easily in the normal scrotum. CAVD is a distinct clinical entity. The diagnosis is easily made on scrotal examination [3]. A lower frequency of CFTR mutations has been detected in patients with CUAVD [4]. Between $11 \%$ and $26 \%$ of patients with CAVD have renal agenesis [5]. The association of CAVD with renal agenesis in cryptorchidism is very low. CFTR mutations are detected in men with CBAVD and, with a lesser frequency in men with CUAVD, it appears that a normal amount of functional CFTR protein is required to ensure proper development of the vas deferens. However the lack of CFTR mutations detected in CAVD with renal anomalies also suggest that the mesonephric duct plays a role in the development of the vas deferens. CAVD is the cause of male infertility and hence genetic counseling plays a major role in patients with CAVD [5]

\section{Conclusions}

CUAVD associated with renal anomalies and cryptorchidism is a rare diagnosis. CUAVD may be associated with cystic fibrosis and evaluation of the same needs to be considered in all such patients. CAVD can cause infertility in men.Genetic counseling plays a major role in patients with CAVD because of its intricate relations with CFTR gene.

\section{References}

1 Mickle, J., Milunsky, A., Amos, J.A. and Oates, R.D. Congenital unilateral absence of the vas deferens: a heterogeneous disorder with two distinct subpopulations based upon aetiology and mutational status of the cystic fibrosis gene. Hum.Reprod. 1995; 10:1728-1735.

2 Holsclaw, D.S., Perlmutter, A.D., Jockin, H. and Shwachman, H. Genital abnormalities in male patients with cystic fibrosis. J. Urol. 1971; 106: 568-574.

3 Marc De Braekeleer and Claude Ferec. Mutations in the cystic fibrosis gene in men with congenital bilateral absence of the vas deferens. Molecular Human Reproduction 1996; 2(9):669-677.

4 Casals, T, Bassas, L1, Ruiz Romero, J et al. Extensive analysis of 40 infertile patients with congenital absence of vas deferens: in $50 \%$ of cases only one CFTR allele could be detected. Hum. Genet. 1995; 95:205-211.

5 Schelgel, P.N., Shin, D. and Goldstein, M. Urogenital anomalies in men with congenital absence of the vas deferens. J. Urol. 1996; 155:1644-1648.

\section{Key points:}

- CUAVD can be associated with renal anomalies and cryptorchidism is a rare diagnosis.

- CAVD can be a cause of infertility in males. 